\title{
L'ÉMERGENCE DU CONCEPT D'ENTREPRISE SOCIALE
}

\author{
Jacques Defourny
}

De Boeck Université | Reflets et perspectives de la vie économique

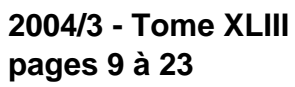

ISSN 0034-2971

Article disponible en ligne à l'adresse:

http://www.cairn.info/revue-reflets-et-perspectives-de-la-vie-economique-2004-3-page-9.htm

Pour citer cet article :

Defourny Jacques , « L'émergence du concept d'entreprise sociale »,

Reflets et perspectives de la vie économique, 2004/3 Tome XLIII, p. 9-23. DOI : 10.3917/rpve.433.0009

Distribution électronique Cairn.info pour De Boeck Université.

(C) De Boeck Université. Tous droits réservés pour tous pays.

La reproduction ou représentation de cet article, notamment par photocopie, n'est autorisée que dans les limites des conditions générales d'utilisation du site ou, le cas échéant, des conditions générales de la licence souscrite par votre établissement. Toute autre reproduction ou représentation, en tout ou partie, sous quelque forme et de quelque manière que ce soit, est interdite sauf accord préalable et écrit de l'éditeur, en dehors des cas prévus par la législation en vigueur en France. II est précisé que son stockage dans une base de données est également interdit. 


\title{
L'émergence du concept d'entreprise sociale
}

Jacques Defourny*

\begin{abstract}
This paper tries to show how the concept of social enterprise is increasingly used in many countries. In Europe, the most significant steps have been taken by the Italian parliament in the early 90's and since 2002 by the UK government which has decided to map and promote social enterprises. From a scientific point of view, the approach built by the EMES European Research Network, based on comparative research in all EU countries, provides a solid basis for further theoretical and empirical work as well as new insights into the whole social economy.
\end{abstract}

Keywords - Social enterprises, entrepreneurship, non-profit organizations, social economy, social cooperatives.

Alors que pratiquement personne ne s'y référait il y a encore une douzaine d'années, la notion d'entreprise sociale est en train d'opérer une étonnante percée des deux côtés de l'Atlantique. Aux États-Unis, elle a trouvé un premier écho significatif au début des années 1990 : parmi d'autres repères possibles, on peut citer la "Social Enterprise Initiative ", lancée en 1993 par la Harvard Business School. Depuis lors, d'autres grandes universités (Columbia, Yale, etc.) ainsi que diverses fondations ont mis sur pied des programmes de formation et de soutien aux entreprises sociales.

Dans le contexte américain, l'idée d'entreprise sociale est néanmoins restée fort large et souvent assez vague, désignant principalement des activités économiques marchandes mises au service d'un but social ${ }^{1}$. L'entreprise sociale est

\footnotetext{
* Jacques DEFOURNY est professeur au Département d'économie et directeur du Centre d'Économie Sociale à l'Université de Liège ; il est également le coordinateur du réseau européen EMES.

1. Selon la "Social Enterprise Alliance » créée en 2002 par diverses fondations et organisations de soutien, on peut appeler « entreprise sociale » " any earned-income business or strategy undertaken by a nonprofit to generate revenue in support of its charitable mission ». Cette vision se retrouve aussi par exemple dans les divers programmes de la NESsT (Nonprofit Enterprise and Selfsustainability Team). Quant au « Social Enterprise Knowledge Network » formé en 2001 autour de la Harvard Business School, il se voudrait plus précis mais il élargit encore le champ : "a social enterprise is any kind of enterprise and undertaking, encompassed by nonprofit organisations, forprofit companies or public sector businesses engaged in activities of significant social value or in the production of goods and services with a embedded social purpose ". Voir aussi le Social Enterprise Magazine Online (www.socialenterprisemagazine.org).
} 
alors vue comme une réponse novatrice aux problèmes de financement des organisations « non-profit », lesquelles se heurtent de plus en plus à des limites dans la collecte de dons privés ou dans leur recherche de subsides auprès des pouvoirs publics et des fondations. Le concept est aussi utilisé pour souligner le caractère innovant et le risque financier assumé par certains types d'initiatives².

En Europe, c'est aussi au début des années 1990 que le concept a fait son apparition et ce, sous une impulsion d'abord italienne. En 1991 en effet, une loi a été votée par le Parlement italien offrant un statut spécifique aux " coopératives sociales » qui se sont alors développées de manière très impressionnante. Cellesci sont nées surtout pour répondre à une série de besoins non satisfaits ou mal rencontrés par les services publics ${ }^{3}$. Ces nouvelles dynamiques entrepreneuriales à finalité sociale ont été observées et analysées dès 1990 par une revue baptisée «Empresa sociale».

Dans un second temps, constatant des initiatives analogues, quoique d'ampleur nettement moindre, dans divers autres pays européens, un réseau européen de chercheurs s'est constitué en 1996 pour étudier l'émergence des entreprises sociales en Europe. Couvrant l'ensemble des quinze pays qui formaient alors l'Union européenne, ce réseau a travaillé pendant quatre ans et a progressivement élaboré une approche commune de l'entreprise sociale ${ }^{4}$.

Plus récemment, une brusque accélération s'est produite au Royaume-Uni : en 2002, le gouvernement de T. Blair a lancé une « Coalition for Social Enterprise » et créé une "Social Enterprise Unit » pour améliorer la connaissance et surtout promouvoir les entreprises sociales dans le pays tout entier. Dans le même cadre, la Secrétaire au Commerce et à l'Industrie, qui a la tutelle de cette Unité, a proposé sa propre définition de l'entreprise sociale et un premier inventaire des entreprises sociales a été réalisé récemment.

Avant de présenter ces conceptions de l'entreprise sociale, il est utile de souligner que celle-ci peut être envisagée dans une double signification : d'une part, elle désigne de nouvelles organisations, des entités crées "ex nihilo » que l'on peut considérer comme un sous-ensemble de l'économie sociale ; d'autre part, elle peut aussi désigner un processus, un nouvel esprit entrepreneurial qui touche et refaçonne des expériences plus anciennes de ce troisième secteur. En ce sens, loin de se substituer aux conceptions existantes de ce dernier, l'approche de l'entreprise sociale présentée ci-après doit plutôt être vue comme un éclairage susceptible d'enrichir ces dernières et de mettre en lumière certaines dynamiques particulières au sein de l'économie sociale ou du «non-profit sector ».

2. Voir par exemple Dees (1998) et Young (2001).

3. Borzaga \& Santuari (2001).

4. Voir Borzaga \& Defourny (2001). Dans le monde francophone, il est arrivé que le terme « entreprise sociale " soit utilisé par facilité pour désigner les entreprises et les organisations de l'économie sociale. II semble que la première publication scientifique se référant à cette notion soit due à la Fondation Roi Baudouin qui souhaitait voir explorée une forme d'entreprise visant l'insertion professionnelle durable de personnes marginalisées sur le marché du travail (Defourny, 1994). Cet ouvrage intitulé "Développer l'entreprise sociale " a servi de base pour des expériences pilotes puis la reconnaissance de ce qu'on appelle aujourd'hui « entreprises d'insertion » en Wallonie et à Bruxelles et « invoegbedrijven » en Flandre. 


\section{UN NOUVEL ENTREPRENEURIAT SOCIAL}

Pourquoi parler d'un nouvel entrepreneuriat social et non simplement d'un développement d'organisations «non-profit » ou d'économie sociale ? Cette question renvoie directement aux diverses théories de l'entrepreneuriat que l'on ne prétend pas ici passer en revue. Nous nous contenterons de nous référer à l'œuvre classique de J. Schumpeter pour lequel le développement économique est un « process of carrying out new combinations » dans le processus de production ${ }^{5}$. Selon lui, les entrepreneurs sont précisément les personnes dont la fonction est de mettre en œuvre ces nouvelles combinaisons. L'entrepreneur n'est pas nécessairement propriétaire d'une entreprise mais, pour Schumpeter, il apporte le changement sur au moins un des plans suivants: I'introduction d'un nouveau produit ou d'une nouvelle qualité de produit, l'introduction d'une nouvelle méthode de production, l'ouverture d'un nouveau marché, la conquête d'une nouvelle source de matières premières ou l'organisation renouvelée d'une branche d'activités.

À la suite des travaux de D. Young (1983) et de Ch. Badelt (1997), on peut adapter cette typologie au troisième secteur et, pour chacun de ces plans, se demander dans quelle mesure on peut identifier un nouvel entrepreneuriat au sein de l'économie sociale.

\subsection{Nouveaux produits ou nouvelles qualités de produit}

De nombreuses analyses du troisième secteur ont déjà montré que celui-ci s'est souvent développé pour répondre à des besoins auxquels le secteur privé classique ou l'État ne pouvaient donner une réponse satisfaisante ${ }^{6}$. Innombrables sont les exemples d'organisations qui ont inventé de nouveaux types de services pour relever les défis de leur temps. En cela, on peut dire qu'aujourd'hui comme hier, beaucoup d'entre elles naissent ou sont nées d'une dynamique entrepreneuriale. Mais y a-t-il quelque chose de spécifique aux deux dernières décennies ? Nous pensons pouvoir parler d'un nouvel entrepreneuriat, davantage sans doute en Europe qu'aux États-Unis, parce que la crise de nos États-providence (en termes de budget, d'efficacité et de légitimité) a fait que les pouvoirs publics ont de plus en plus laissé à l'initiative privée des réponses qu'ils auraient organisées euxmêmes si la conjoncture avait été celle des Trente Glorieuses. Le cas du RoyaumeUni est sans doute le plus frappant, mais la plupart des États membres de l'Union européenne ont connu à des degrés divers la même tendance. Ainsi, les deux champs privilégiés par les travaux du Réseau EMES, celui de l'insertion professionnelle des chômeurs peu qualifiés et celui des services aux personnes, ont vu de multiples innovations en termes de création d'activités ou en termes de services mieux adaptés aux besoins, que ce soit dans la formation par le travail, l'ac-

5. Schumpeter (1934), p. 66.

6. C'est même là un thème majeur de la littérature qui cherche à identifier les principales raisons de l'existence du troisième secteur. 
cueil de la petite enfance, les services aux personnes âgées ou encore l'aide à certaines catégories défavorisées (enfants maltraités, réfugiés, immigrés...) ${ }^{7}$.

Cet entrepreneuriat apparaît d'autant plus novateur que, même au sein du troisième secteur, il tranche avec le comportement assez bureaucratique et peu innovant de certaines grandes organisations traditionnelles (par exemple les très grandes fédérations de services sociaux - Wolhlfahrtsverbände - en Allemagne).

\subsection{Nouvelles méthodes d'organisation et/ou de production}

II est courant de voir l'économie sociale organiser ses activités selon des modalités différentes de celles des secteurs privé ou public traditionnels. Mais ce qui frappe le plus dans les générations actuelles d'entreprises sociales, c'est l'implication de différents partenaires, de plusieurs catégories d'acteurs : travailleurs salariés, bénévoles, usagers, organismes d'appui et pouvoirs publics locaux se retrouvent souvent associés dans un même projet, alors que les organisations classiques de l'économie sociale ont généralement été fondées par des groupes sociaux plus homogènes ${ }^{8}$. Si cela ne révolutionne pas nécessairement le processus de production au sens strict, les modes d'organisation d'une activité s'en trouvent souvent transformés. On pourra même parfois parler d'une construction conjointe de l'offre et de la demande quand prestataires et usagers collaborent dans l'organisation et la gestion de certains services de proximité. La mise sur pied de "crèches parentales », en France ou en Suède, constitue une illustration parmi d'autres de telles collaborations.

\subsection{Nouveaux facteurs de production}

Une des spécificités majeures mais anciennes du troisième secteur est sa capacité à mobiliser du travail bénévole. En soi, le recours à du bénévolat n'est donc pas innovant, mais il l'est dans de nombreuses initiatives récentes parce qu'il permet de produire des biens ou services qui n'étaient pas offerts auparavant ou qui l'étaient seulement à l'aide de travail rémunéré (auquel cas il est rare qu'il s'agisse vraiment du même «produit »).

On notera aussi que le bénévolat a profondément changé de nature au cours des dernières décennies : il apparaît beaucoup moins caritatif qu'il y a quarante ou cinquante ans, mais aussi moins « militant » que dans les années 1960 ou 1970. Assez pragmatique, le bénévole d'aujourd'hui est plus orienté vers des finalités " productives », vers des activités répondant à des besoins ciblés. II n'est d'ailleurs

7. Sur l'insertion, voir Defourny, Favreau \& Laville (1998) ou encore Davister, Defourny \& Grégoire (2004) ; sur les services aux personnes, voir Laville \& Nyssens (2001b).

8. Cette plus grande homogénéité se reflète notamment dans les dénominations des différents types de coopératives ou de mutuelles : coopératives de travailleurs, coopératives agricoles, mutuelles de fonctionnaires, d'artisans, d'agriculteurs, etc. 
pas rare que la fonction entrepreneuriale au sens le plus habituel du terme (lancement de l'activité) soit assurée par des bénévoles.

Quant au travail rémunéré, il a lui aussi fait l'objet d'innovations en sens divers. D'une part, beaucoup d'organisations du troisième secteur se sont retrouvées aux avant-postes dans l'expérimentation de formes d'emploi atypiques : engagement de personnel dans le cadre de programmes de résorption du chômage, développement de formules de semi-volontariat ou de travail à temps très partiel, etc. ${ }^{9}$ D'autre part, on peut dire que les statuts classiques des travailleurs sont souvent " enrichis " lorsque ces derniers sont reconnus comme membres à part entière des instances souveraines de l'entreprise sociale, avec le pouvoir de contrôle et de décision qui en découle ${ }^{10}$.

\subsection{Nouveaux rapports au marché}

Dans un nombre croissant de pays, la tendance est aussi au « contracting out » et au développement de quasi-marchés pour certains services auparavant assurés par les pouvoirs publics ou par des organismes privés sans but lucratif privilégiés de longue date par l'État. En effet, dans un souci de réduction des coûts et de meilleure adaptation des prestations aux demandes des usagers, l'autorité publique adopte de plus en plus des systèmes d'appels d'offre. Ceux-ci mettent en concurrence différents types de prestataires pour l'obtention de financements publics liés à la réalisation de cahiers de charges préalablement définis. Ce sont les gouvernements conservateurs anglais qui sont allés le plus loin dans cette direction, mais ce passage d'une "régulation tutélaire » à une "régulation concurrentielle » est un peu partout dans l'air du temps.

Des mutations aussi profondes de l'État-providence ne peuvent qu'avoir des conséquences importantes à différents niveaux. Pour notre propos, contentonsnous de souligner ce qui tend à accentuer le caractère entrepreneurial des associations, au sens d'une ressemblance plus marquée avec les entreprises classiques et aussi, en partie, en termes de «nouvelles combinaisons » schumpéteriennes ${ }^{11}$ :

- les associations déjà en place se retrouvent dans une logique de concurrence, y compris parfois avec des entreprises à but lucratif, pour leurs réponses aux appels d'offre ;

- $\quad$ elles sont par là même contraintes d'installer ou de renforcer en leur sein une culture gestionnaire largement calquée sur celle du monde marchand ;

- la rupture de certains monopoles publics (par exemple en Suède) ou de monopoles de grandes fédérations nationales de services sociaux (par exemple

9. On se borne ici à constater ces évolutions sans prétendre évidemment qu'il s'agit d'avancées sociales.

10. À nouveau, il faut rester prudent dans l'interprétation de cette évolution : une partie de ce comportement innovant vient des organisations elles-mêmes, mais il s'agit aussi de réactions et d'adaptations à des impulsions ou à des restrictions inhérentes à des politiques publiques.

11. Voir, à ce sujet, Laville et Sainsaulieu (1997). 
en Allemagne) stimule l'apparition de nouvelles initiatives privées (avec ou sans but de lucre) d'emblée profilées en fonction de ce contexte ;

- last but not least, tant pour les anciennes que pour les nouvelles associations, il y a un accroissement du risque économique puisque leur financement est désormais tributaire de leur capacité à capter ces quasi-marchés et à satisfaire les usagers.

Enfin, il va de soi que joue dans le même sens le développement d'une demande privée solvable pour certains services qui sont rendus accessibles par l'élévation continue du niveau de vie d'une partie importante de la population. Ainsi par exemple, les personnes âgées qui bénéficient d'allocations de pension confortables ou qui ont accumulé une épargne substantielle représentent de nouveaux marchés, mais ceux-ci sont souvent très concurrentiels.

\subsection{Nouvelles formes d'entreprises}

Comme pour confirmer qu'on est bien en présence d'un entrepreneuriat plutôt inédit, diverses législations nationales ont construit des cadres juridiques nouveaux, censés être mieux adaptés à ce type d'initiatives que les moules associatifs ou coopératifs traditionnels.

On a déjà évoqué le statut voté en 1991 pour les coopératives sociales italiennes. En 1995, la Belgique a institué la "société à finalité sociale » (SFS) tandis qu'en mai 1999, le Portugal a mis au point un statut de "coopérative sociale à responsabilité limitée ». De même, la loi générale espagnole de 1999 sur les coopératives prévoit désormais une place pour les coopératives de services sociaux et des cadres juridiques précis ont été définis au niveau de différentes régions. Plus récemment encore, la France a créé le statut de « société coopérative d'intérêt collectif » (SCIC) et un nouvelle législation instituant la "community interest company » est sur le point d'être adoptée au Royaume-Uni.

D'une manière générale, ces nouveaux cadres juridiques visent à encourager les dynamiques entrepreneuriales et commerciales qui s'inscrivent dans un projet social. Ils peuvent aussi permettre de formaliser le caractère " multi-stakeholders » de nombreuses initiatives en associant au contrôle de celles-ci les différentes parties prenantes (travailleurs rémunérés, bénévoles, usagers...). Toutefois, on soulignera qu'en dehors de l'Italie, une grande partie des entreprises sociales se retrouvent encore sous les statuts classiques du troisième secteur.

\section{VERS UNE DÉFINITION DE L'ENTREPRISE SOCIALE}

À l'heure actuelle, il semble bien que seules deux définitions de l'entreprise sociale émergent de manière consistante et constituent une référence conceptuelle pour un ensemble d'autres travaux : celle forgée par le Réseau européen EMES et celle avancée par le gouvernement britannique en 2002. 
Envisageons d'abord cette dernière. Dans le cadre d'un document rendu public en juillet 2002 et intitulé "Social Enterprise : A Strategy for Success », la Secrétaire au Commerce et à l'Industrie P. Hewitt a proposé la définition suivante: " une entreprise sociale est une activité commerciale (business) ayant essentiellement des objectifs sociaux et dont les surplus sont principalement réinvestis en fonction de ces finalités dans cette activité ou dans la communauté, plutôt que d'être guidés par le besoin de maximiser les profits pour des actionnaires ou des propriétaires ${ }^{12}$. Dans la foulée, toute une série de travaux empiriques ont été réalisés à travers le pays sur la base de cette définition alors que, jusque là, une grande variété d'approches co-existaient autour de cette notion de plus en plus à la mode. Un travail de synthèse a ensuite été confié à un bureau d'études ${ }^{13}$ qui a fait rapport à la "Social Enterprise Unit » en juillet 2003. Ce rapport note que la définition du gouvernement a apporté plus de clarté, mais il relève aussi une série de difficultés en termes de cohérence et de comparabilité pour la rendre opérationnelle. Pour pallier ces insuffisances, ECOTER note qu'un certain nombre d'études ont examiné en plus la manière dont ces entreprises fonctionnent effectivement. À leur suite, il estime que la définition doit être décomposée en une série de caractéristiques, ou indicateurs, qui peuvent, mieux qu'un overall statement, être utilisées pour inventorier les entreprises sociales au Royaume-Uni. De tels indicateurs relèveraient de trois registres principaux : l'orientation entrepreneuriale, les objectifs sociaux et la propriété sociale des activités observées ${ }^{14}$.

En d'autres termes, si l'approche britannique mérite d'autant plus l'attention qu'elle fonde une politique promouvant explicitement l'entreprise sociale, il semble qu'elle soit encore en pleine construction. En outre, comme les travaux qu'elle suscite se situent jusqu'à présent dans un cadre national très circonscrit, il nous paraît raisonnable de privilégier à ce stade l'approche développée depuis la seconde moitié des années 1990 par le Réseau européen EMES ${ }^{15}$. Celle-ci est en effet le fruit d'un long travail de dialogue entre plusieurs disciplines (économie, sociologie, science politique et management), mais aussi entre les diverses traditions et sensibilités nationales au sein de l'Union européenne. Enfin, orientée par un projet à la fois théorique et empirique, elle a d'emblée privilégié l'identification et l'explicitation d'indicateurs à l'élégance d'une définition bien ramassée.

Dès 1996, le Réseau EMES s'est attelé à définir des critères communs pour repérer les réalités susceptibles d'être appelées « entreprises sociales » dans chacun des quinze pays étudiés. C'est ainsi qu'une working definition a été construite qui était surtout une hypothèse de travail et qui ne prétendait pas cerner toute la

12. Hewitt (2002).

13. ECOTER Research \& Consultants Limited.

14. ECOTER (2003), pp. 22-26. On notera aussi que cette étude avance, comme première estimation, le chiffre de 5300 entreprises sociales au Royaume-Uni.

15. EMES est l'acronyme du titre français de ce vaste projet de recherche sur "l'émergence des entreprises sociales en Europe " (1996-1999). Désignant d'abord le réseau de chercheurs aux yeux de son commanditaire, la DG Recherche de la Commission européenne, ce nom a été conservé lorsque le réseau a poursuivi divers autres projets sur les entreprises sociales et l'ensemble de l'économie sociale. II rassemble aujourd'hui neuf centres de recherche spécialisés en ces matières et quelques chercheurs individuels à travers toute l'Europe. 
réalité des entreprises sociales. À l'expérience toutefois, ce faisceau d'indicateurs s'est révélé constituer un cadre conceptuel robuste et fiable.

Cette approche est fondée sur deux séries d'indicateurs, ou critères, les uns plutôt économiques, les autres à dominante sociale. Les voici tels que présentés dans les travaux d'EMES ${ }^{16}$.

Pour attester le caractère économique et entrepreneurial des initiatives envisagées, quatre éléments ont été retenus :

a) Une activité continue de production de biens et / ou de services.

Les entreprises sociales, à l'inverse de certaines organisations non-profit traditionnelles, n'ont normalement pas comme activité principale la défense d'intérêts, ni la redistribution d'argent (comme c'est le cas, par exemple, de beaucoup de fondations), mais elles sont directement impliquées, d'une manière continue, dans la production de biens et / ou l'offre de services aux personnes. L'activité productive représente donc la raison d'être - ou l'une des principales raisons d'être - des entreprises sociales.

b) Un degré élevé d'autonomie.

Les entreprises sociales sont créées par un groupe de personnes sur base d'un projet propre et elles sont contrôlées par celles-ci. Elles peuvent dépendre de subsides publics mais ne sont pas dirigées, que ce soit directement ou indirectement, par des autorités publiques ou d'autres organisations (fédérations, entreprises privées...). Elles ont le droit tant de faire entendre leur voix ("voice ») que de mettre un terme à leurs activités (" exit »).

c) Un niveau significatif de prise de risque économique.

Les créateurs d'une entreprise sociale assument totalement ou partiellement le risque qui y est inhérent. À l'inverse de la plupart des institutions publiques, leur viabilité financière dépend des efforts consentis par leurs membres et par leurs travailleurs pour assurer à l'entreprise des ressources suffisantes.

d) Un niveau minimum d'emploi rémunéré.

Tout comme les organisations "non-profit » traditionnelles, les entreprises sociales peuvent faire appel à des ressources tant monétaires que non monétaires, et à des travailleurs rémunérés comme à des volontaires. Cependant, l'activité de l'entreprise sociale requiert un niveau minimum d'emploi rémunéré.

Pour identifier la dimension sociale des initiatives, cinq indicateurs ont été privilégiés:

e) Un objectif explicite de service à la communauté.

L'un des principaux objectifs des entreprises sociales est le service à la communauté ou à un groupe spécifique de personnes. Dans la même perspective, une caractéristique des entreprises sociales est constituée par leur volonté de promouvoir le sens de la responsabilité sociale au niveau local.

16. Defourny (2001), pp. 16-18. Les premiers travaux publiés par EMES datent de 1999, mais l'ensemble de ces indicateurs étaient déjà identifiés dans des rapports intermédiaires (EMES European Network, 1997 et 1998) qui ont notamment été utilisés par l'OCDE (1999). 
f) Une initiative émanant d'un groupe de citoyens.

Les entreprises sociales résultent d'une dynamique collective impliquant des personnes qui appartiennent à une communauté ou à un groupe qui partage un besoin ou un objectif bien défini; cette dimension est maintenue dans le temps d'une manière ou d'une autre, même s'il ne faut pas négliger l'importance d'un leadership souvent exercé par une personne ou un noyau restreint de dirigeants.

g) Un pouvoir de décision non basé sur la détention de capital.

Ce critère renvoie généralement au principe "un membre, une voix », ou tout au moins à un processus de décision dans lequel les droits de vote au sein de l'assemblée détenant le pouvoir de décision ultime ne sont pas répartis en fonction d'éventuelles participations au capital. En outre, si les propriétaires du capital social sont importants, le pouvoir de décision est généralement partagé avec d'autres acteurs.

h) Une dynamique participative, impliquant différentes parties concernées par l'activité.

La représentation et la participation des usagers ou des clients, l'exercice d'un pouvoir de décision par diverses parties prenantes au projet et une gestion participative constituent souvent des caractéristiques importantes des entreprises sociales. Dans bon nombre de cas, l'un des objectifs des entreprises sociales est de promouvoir la démocratie au niveau local par le biais de l'activité économique.

i) Une limitation de la distribution des bénéfices.

Si les entreprises sociales peuvent être des organisations caractérisées par une obligation absolue de non-distribution des bénéfices, elles peuvent aussi être des organisations qui, comme les coopératives dans beaucoup de pays, ont le droit de distribuer des bénéfices, mais de manière limitée - ce qui permet d'éviter un comportement visant à la maximisation du profit.

\section{DU BON USAGE DE L'APPROCHE «EMES "}

Pour être comprise correctement et éventuellement utilisée, l'approche du Réseau EMES doit intégrer deux considérations supplémentaires dont la première a déjà été soulignée : ces indicateurs économiques et sociaux peuvent à la fois permettre d'identifier de toutes nouvelles entreprises sociales et également amener à désigner comme entreprises sociales des organisations plus anciennes reconfigurées par de nouvelles dynamiques internes.

Un autre élément s'avère encore plus fondamental : les indicateurs qui viennent d'être énumérés ne forment pas l'ensemble des conditions qu'une organisation doit remplir pour pouvoir être qualifiée d'entreprise sociale. Plutôt que de constituer des critères normatifs, ces indicateurs décrivent plutôt un « idéal-type » permettant de se situer au sein de la galaxie des entreprises sociales. Un peu à l'instar d'une boussole ${ }^{17}$, un tel outil peut aider le chercheur à situer les entités

17. Se diriger dans une direction Nord-Est ou Nord-Ouest n'est en soi ni mieux ni moins bien que de marcher plein Nord. 
observées les unes par rapport aux autres et éventuellement à tracer les limites de l'ensemble qu'il considérera comme celui des entreprises sociales. Ainsi par exemple, dans leur première phase d'identification, les membres du Réseau EMES avaient-ils distingué un ensemble « central » et un ensemble plus "périphérique » d'entreprises sociales autour de cet idéal-type.

Avec de telles précautions, l'approche EMES peut s'avérer féconde sur le plan empirique. Ainsi, en étudiant 151 organismes primés depuis vingt ans par la Fondation Crédit Coopératif en France, J.-F. Draperi (2003) a retrouvé à des degrés divers la plupart des traits esquissés ci-dessus. Alors qu'il ne pensait pas au départ conclure sur l'entreprise sociale, c'est pourtant ce qu'il fait finalement en se référant à l'approche d'EMES et en soulignant la capacité d'innovation sociale de ces organismes.

Dans la même ligne, les chercheurs d'EMES viennent de réaliser un inventaire des différents types d'entreprises sociales dans le champ de la formation par le travail et de la réinsertion professionnelle de personnes peu qualifiées. Portant sur douze pays de l'UE, ce repérage a combiné les indicateurs présentés ci-avant avec des critères propres à ce champ pour forger la notion d' "entreprise sociale d'insertion " (ESI) ou work integration social enterprise (WISE). Un tel cadre conceptuel a permis d'identifier pas moins de 39 catégories ou modèles d'ESI au sein des douze pays concernés ${ }^{18}$.

\section{QUELQUES ILLUSTRATIONS À TRAVERS L'UNION EUROPÉENNE}

Dans leur première recherche (1996-1999) ${ }^{19}$, qui couvrait la totalité des pays de I'UE, les chercheurs du Réseau EMES ont construit l'approche décrite à la section 3 puis se sont employés, sur cette base, à décrire et à analyser les entreprises sociales émergeant dans un ou plusieurs des trois champs suivants : la formationréinsertion par le travail, les services aux personnes et le développement local.

La frontière entre la prestation de services sociaux et les activités visant la réinsertion de personnes exclues du marché de l'emploi est relativement floue. Dans plusieurs pays en effet, le travail social ou les services pour les personnes atteintes de handicap ou confrontées à d'autres difficultés ont évolué vers des activités plus productives. Dans de nombreux cas, il n'y a pas non plus de délimitation claire entre les entreprises sociales visant l'insertion par le travail de personnes atteintes de handicaps et celles s'adressant aux personnes socialement exclues (toxicomanes, prisonniers, jeunes ayant abandonné l'école...). Bon nombre d'entreprises sociales offrent des emplois stables mais beaucoup d'autres doivent être considérées comme liées aux «marchés du travail transitionnels »; en d'autres

18. Les travaux nationaux ont été publiés dans la collection des EMES Working Papers. Ils sont aussi consultables sur le site www.elexies.info. Pour une synthèse, voir Spear \& Bidet (2003) ainsi que Davister, Defourny \& Grégoire (2003).

19. Borzaga \& Defourny (2001). 
termes, ces entreprises constituent une étape sur le parcours de réinsertion vers le marché du travail classique. Enfin, soulignons aussi la participation des entreprises sociales aux dynamiques de développement local dans les zones défavorisées.

\section{JALONS POUR UNE THÉORIE DE L'ENTREPRISE SOCIALE}

Dans la dernière phase de sa recherche fondatrice, le Réseau EMES a présenté quelques premiers pas pour l'élaboration progressive d'une théorie spécifique de l'entreprise sociale ${ }^{20}$. Ainsi, Bacchiega et Borzaga (2001) ont utilisé les outils offerts par la théorie institutionnelle des organisations pour mettre en lumière la nature innovante des entreprises sociales. En outre, les traits définissant l'entreprise sociale sont interprétés comme formant un système d'incitants original tenant compte des objectifs, potentiellement conflictuels, poursuivis par les différentes catégories de parties prenantes (stakeholders). De son côté, Evers (2001) a développé une analyse plus sociopolitique pour montrer qu'une telle structure «mulistakeholder - multiple goal » peut être mieux comprise en faisant appel à la notion de « capital social ». Pour lui, la production de capital social peut aussi devenir un objectif explicite d'organisations comme les entreprises sociales. Quant à Laville et Nyssens (2001), ils ont proposé des éléments pour une théorie intégrée d'un « idéal-type » de l'entreprise sociale, combinant les dimensions économiques, sociales et politiques de celle-ci. Eux aussi insistent sur le rôle du capital social, mobilisé et reproduit sous des formes spécifiques. En outre, ils mettent en évidence la nature particulièrement hybride et composite des ressources de l'entreprise sociale et y voient un atout essentiel de celle-ci pour résister aux tendances à « l'isomorphisme institutionnel » qui menacent toutes les organisations de l'économie sociale. Enfin, Borzaga et Solari (2001) ont exploré les principaux défis de management auxquels sont confrontés les dirigeants et les membres d'entreprises sociales. Ils se sont aussi interrogés sur les modèles de gouvernance éventuellement spécifiques à celles-ci.

Sur le plan théorique, on peut également voir dans le concept d'entreprise sociale le vecteur d'une approche plus intégrée de toute l'économie sociale. En effet, l'appréhension de celle-ci souffre régulièrement de deux tensions difficiles à dépasser. La première est celle provenant de l'écart existant entre des entreprises offrant toute leur production sur le marché (comme la plupart des coopératives en Belgique) et des associations dont les activités apparaissent peu économiques (par exemple celles d'un mouvement de jeunes) et dont les ressources sont entièrement non marchandes, voire non monétaires. Une deuxième tension existe entre les organisations dites d'intérêt mutuel (coopératives, mutuelles et un grand nombre d'associations) qui visent à servir leurs membres et celles dites d'intérêt général qui se mettent au service d'une collectivité plus large (par exemple, dans la lutte contre la pauvreté et l'exclusion, la coopération au développement, la protection de l'environnement, etc.).

20. Voir Borzaga \& Defourny (2001). 
Ces deux tensions peuvent être en partie illustrées par le graphique ci-après. La première y est figurée par la coexistence de deux sphères très distinctes : d'un côté, celle de la tradition coopérative qui a généré une littérature et des écoles de pensée bien spécifiques ; de l'autre, celle des initiatives et des mouvements associatifs qui, eux aussi, ont mobilisé de nombreux sociologues et politologues et trouvé une vigueur particulière dans le courant américain des non-profit organizations. La seconde tension se représente plus difficilement mais se retrouve à l'intérieur de chacune des deux sphères où les organisations d'intérêt mutuel sont plutôt localisées aux extrémités gauche et droite du graphique tandis que les organisations d'intérêt général se situent davantage vers le centre.

Le rôle intégrateur du concept d'entreprise sociale réside avant tout en ce qu'il crée une force d'attraction réciproque entre les deux sphères. II le fait en se fixant, au sein de chaque sphère, sur des organisations qu'il attire et englobe d'un seul tenant, parce qu'elles sont finalement très proches, le choix d'un statut coopératif ou associatif dépendant surtout des dispositifs juridiques offerts par les législations nationales.

\section{Schéma 1 - Les entreprises sociales, à la croisée des secteurs coopératif et non-profit}

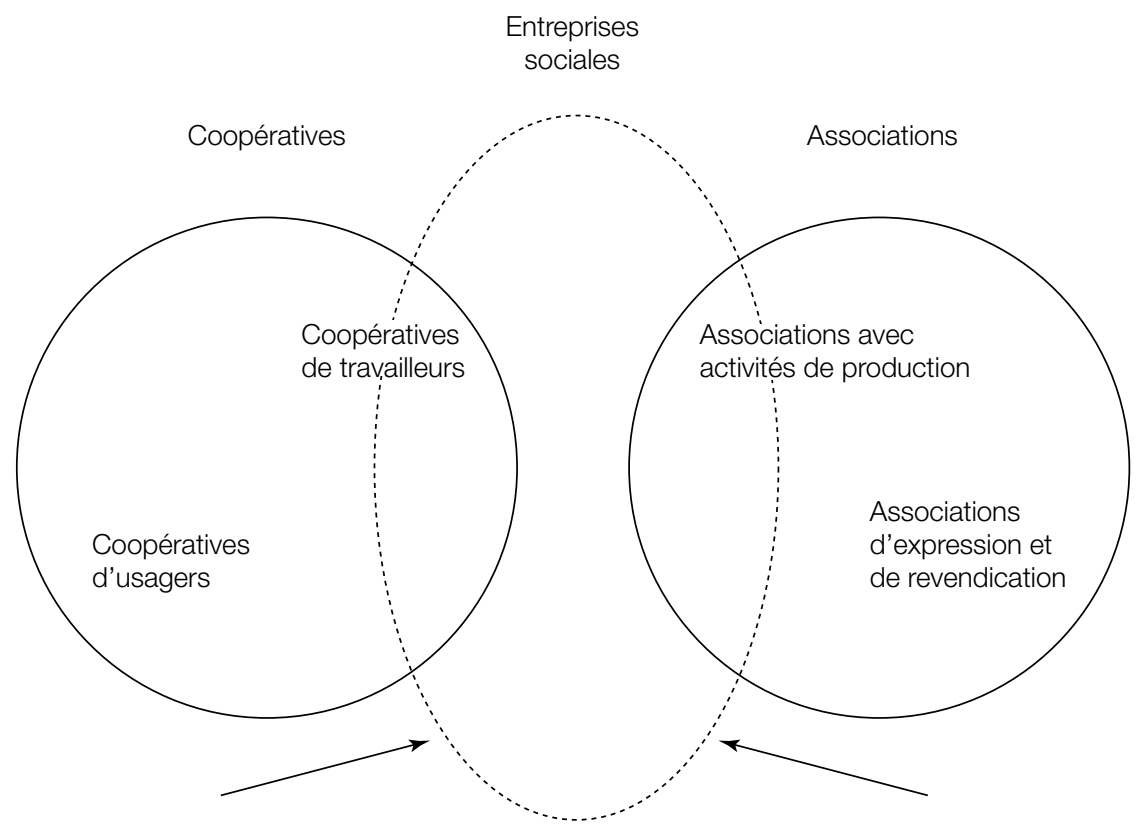

On peut encore noter que les entreprises sociales semblent plus proches des coopératives de travailleurs que des coopératives d'usagers traditionnelles (souvent de très grande taille), comme le démontrent les exemples des labour cooperatives en Finlande, des coopératives agro-touristiques en Grèce ou des 
coopératives de soins à domicile au Royaume-Uni. Cependant, certaines caractéristiques des entreprises sociales les distinguent des coopératives de travailleurs traditionnelles ; ainsi, leur sociétariat rassemble fréquemment différents types de parties prenantes et elles visent davantage le bénéfice de la communauté dans son ensemble.

Enfin, si les entreprises sociales ont essentiellement la forme de coopératives ou d'associations, elles peuvent également se développer dans le cadre d'autres statuts légaux. En Belgique, c'est le cas de certaines sociétés à finalité sociale qui ont opté pour le statut de société anonyme ou de SPRL plutôt que pour le statut coopératif. C'est encore bien plus vrai dans certains autres pays qui ont conçu des cadres juridiques vraiment distincts pour les entreprises sociales, comme la nouvelle community interest company au Royaume-Uni.

\section{CONCLUSION}

Nous espérons avoir montré qu'au lieu de se substituer aux conceptions existantes du troisième secteur ou de leur faire concurrence, la notion d'entreprise sociale vient plutôt enrichir celles-ci, en particulier la notion d'économie sociale qui embrasse depuis toujours les coopératives et les associations ${ }^{21}$. Elle contribue même, comme on vient de le voir, à mettre davantage de « liant » au sein même de l'économie sociale dont les composantes coopératives, mutualistes et associatives sont parfois juxtaposées de manière un peu formelle et statique.

Enfin, si les analyses sociopolitiques de l'économie sociale n'ont jamais manqué, c'est sans doute sur le versant économique de celui-ci qu'il reste le plus à faire. D'importants progrès ont été réalisés ces dernières années sur le plan de la statistique économique ${ }^{22}$. Des pas significatifs ont aussi été accomplis pour appréhender les défis spécifiques du troisième secteur en termes de gestion, comme en témoigne le développement de programmes de formation en la matière. Dans la même veine, le concept d'entreprise sociale apparaît comme une pierre d'angle indispensable à l'édifice: il souligne combien l'innovation sociale implique souvent la prise de risques économiques et que l'entrepreneuriat, si important à promouvoir dans des régions en redéploiement, peut s'exprimer dans des formes plurielles.

21. C'était déjà le cas chez Charles Gide en 1900, comme l'a bien montré A. Gueslin (1987).

22. Notamment dans le cadre de la vaste recherche internationale sur le non-profit sector coordonnée par l'université américaine Johns Hopkins. Pour la Belgique, comment ne pas souligner la caractère pionnier du premier "Compte satellite des institutions sans but lucratif » publié par la Banque Nationale en mars 2004. 


\section{RÉFÉRENCES}

Bacchiega A. \& Borzaga C. (2001), "Social Enterprises as Incentive Structures : an Economic Analysis ", in Borzaga C. \& Defourny J., eds, The Emergence of Social Enterprise, Routledge, pp. 273-294.

Badelt Ch. (1997), "Entrepreneurship Theories of the Non-Profit Sector », Voluntas, vol. $8, n^{\circ} 2$, pp. 162-178.

Borzaga C. \& Defourny J., eds. (2001), The Emergence of Social Enterprise, Routledge, London (paperback edition : 2004).

Borzaga C. \& Santuari A. (2001), "Italy : from Traditional Co-operatives to Innovative Social Enterprises ", in Borzaga C. \& Defourny J., eds, The Emergence of Social Enterprise, Routledge, pp. 166-181.

Borzaga C. \& Solari, L. (2001), "Management Challenges for Social Enterprises », in Borzaga C. \& Defourny J., eds, The Emergence of Social Enterprise, Routledge, pp. 333-349.

Davister C., Defourny J. \& Grégoire O. (2003), «Les entreprises sociales d'insertion dans l'Union européenne : un aperçu général », EMES Working Papers, no 03/11, à paraître dans la Revue des Études Coopératives, Mutualistes et Associatives (RECMA), Paris.

Dees J. G. (1998), «The Meaning of Social Entrepreneurship », Stanford University, miméo.

Defourny, J., ed. (1994), Développer l'entreprise sociale, Bruxelles, Fondation Roi Baudouin.

Defourny J. (2001), "From Third Sector to Social Enterprise », in Borzaga C. \& Defourny J., eds, The Emergence of Social Enterprise, Routledge, pp. 1-28.

Defourny J., Favreau L. \& Laville J.-L., eds (1998), Insertion et nouvelle économie sociale. Un bilan international, Paris, Desclée de Brouwer.

Defourny J. \& Nyssens M. (2001), «Belgium : Social Enterprises in Community Services ", in Borzaga C. \& Defourny J., eds, The Emergence of Social Enterprise, Routledge, pp. 47-64.

Draperi J.-F. (2003), « L'entreprise sociale en France, entre économie sociale et action sociale » in Revue des Études Coopératives, Mutualistes et Associatives (RECMA), $n^{\circ} 288$, pp. 48-66.

ECOTER (2003), "Guidance on Mapping Social Enterprise ", Final Report to the DTI Social Enterprise Unit, London (www.dti.gov.uk/socialenterprise/newsmapping.htm).

EMES Network (1997, 1998, 1999), "The Emergence of Social Enterprises. A New Answer to Social Exclusion in Europe ", Semestrial Progress Reports to the European Commission.

Evers A. (2001), "The Significance of Social Capital in the Multiple Goal and Resource Structure of Social Enterprise ", in Borzaga C. \& Defourny J., eds, The Emergence of Social Enterprise, Routledge, pp. 295-311. 
Evers A. \& Laville J.-L., eds (2004), The Third Sector in Europe, Edward Elgar, Cheltenham.

Gueslin A. (1987), L'invention de l'économie sociale, Paris, Economica.

Hewitt P. (2002), «Social Enterprise. A Strategy for Success », Department of Trade and Industry, London (www.dti.gov.uk/socialenterprise/strategy.htm).

Laville J.-L. \& Nyssens M. (2001), «The Social Enterprise : Towards a Theoretical SocioEconomic Approach », in Borzaga C. \& Defourny J., eds, The Emergence of Social Enterprise, Routledge, pp. 312-332.

Laville J.-L. \& Nyssens M., eds (2001), Les services sociaux entre associations, État et marché, Paris, La Découverte et Syros.

Laville J.-L. \& Sainsaulieu R., eds (1997), Sociologie de l'association, Desclée de Brouwer, Paris.

OCDE (1999), Les entreprises sociales, Programme LEED, OCDE, Paris.

Schumpeter J. A. (1934), The Theory of Economic Development, 3d printing, 1963, New York, Oxford University Press.

Spear R. \& Bidet E. (2003), « The Role of Social Enterprise in European Labour Markets ", EMES Working Papers, n 03/10.

Young D. (1983), If Not for Profit, for What?, Lexington, Mass, Lexington Books.

Young D. (2001), «Social Enterprises in the United States: Alternate Identities and Forms ", Paper presented at the International Conference on Social Enterprise, University of Trento. 